\title{
Characterisation of solution cast cellulose nanofibre - reinforced poly(lactic acid)
}

\author{
D. Y. Liu ${ }^{1}$, X. W. Yuan ${ }^{*}$, D. Bhattacharyya ${ }^{1}$, A. J. Easteal ${ }^{2}$ \\ ${ }^{1}$ Department of Mechanical Engineering, The University of Auckland, Auckland, New Zealand \\ ${ }^{2}$ Department of Chemistry, The University of Auckland, Auckland, New Zealand
}

Received 14 August 2009; accepted in revised form 12 October 2009

\begin{abstract}
Cellulose nanofibres, $20 \mathrm{~nm}$ in diameter and $300 \mathrm{~nm}$ long, were prepared by acid hydrolysis of flax yarns. Composite films containing 2.5 and $5.0 \mathrm{wt} \%$ flax cellulose (FC) fibres were prepared by solution casting of mixtures of poly(lactic acid) (PLA) solution and cellulose nanofibre suspension in chloroform. The resulting composite films and solution cast pure PLA film, with thickness of around $160 \mu \mathrm{m}$, showed good transparency. For composites with 2.5 and $5.0 \mathrm{wt} \% \mathrm{FC}$, the tensile strength increased by 25 and $59 \%$ and tensile modulus by 42 and $47 \%$, respectively, compared to pure PLA film. The composite film with $2.5 \mathrm{wt} \%$ FC combined high strength and ductility with tensile strength of 24.3 $\mathrm{MPa}$ and $70 \%$ elongation at break. Flax cellulose appeared to facilitate nucleation and subsequent crystallisation of PLA more effectively in the amorphous composites than in the crystalline composites.
\end{abstract}

Keywords: polymer composites, poly(lactic acid) (PLA), nanocellulose, biopolymer

\section{Introduction}

Biopolymers are attractive materials because they are biodegradable, recyclable and compostable, and are regarded as the best replacement for conventional packaging materials in respect of energy resources and environmental impact. PLA is a versatile biopolymer made from the renewable agricultural raw material corn, and shows good potential for applications in packaging, and the automotive and biomedical fields. For the packaging industry in particular, PLA has significant potential because of the combination of high clarity and stiffness, and excellent printability, and because it can be manufactured using readily available production technology. PLA is being commercialised as a food packaging polymer for short shelf-life products in common applications such as containers, drinking cups, and overwrap and lamination films [1]. However, the thermal properties and water vapour and gas barrier properties of PLA are inferior to those of conventional petroleum-based polymers [2, 3].

To tailor the properties of PLA it is desirable to combine the polymer with reinforcing elements. Much research is focused on the use of natural fibres as reinforcements for polymer composites, because natural fibres are renewable and abundant [4]. Purified cellulose fibres with one nano-scale dimension have more potential as reinforcing fillers in polymer matrices due to their good mechanical properties with very high bending strength, and stiffness (e.g. Young's modulus of about $150 \mathrm{GPa}$ $[5,6])$. Cellulose in nano-scale has been investigated extensively [7] for preparation of polymer composites and is available from various sources, such as cotton, tunicate, bacteria, ramie and wood. The high aspect ratio and good dispersability of cellulose enhance barrier properties to gases and vapours, because the presence of the impermeable 
crystalline fibres can increase the travel path for gas or vapour movement through the composite (detour factor) and lead to slower diffusion processes $[8,9]$. Thus, preparation of composites with nano-scale fillers has been considered a promising method to improve gas barrier properties and mechanical properties without affecting transparency for packaging applications [10].

In the present work, cellulose fibres, $20 \mathrm{~nm}$ in diameter and $400 \mathrm{~nm}$ long, were obtained from flax yarns by the sulphuric acid hydrolysis method. Pure PLA and its composite films with 2.5 and $5.0 \mathrm{wt} \%$ flax cellulose (FC) were prepared by casting PLA solution, and mixtures of cellulose suspension and PLA solution. The objective of the study was to investigate the effects of addition of nano-FC on the properties of the PLA matrix. The morphology, structure, thermal and mechanical properties are presented in this paper.

\section{Experimental methods}

\subsection{Materials}

Poly(lactic acid) (PLA), 2002 D, was purchased in pellet form from Natureworks Co., Minnetonka, USA. Bleached flax yarns were purchased from Jayashree Textiles, Kolkata, India. Microcrystalline cellulose powder was supplied by SigmaAldrich Inc., St. Louis, USA. Sodium hydroxide was purchased from Ajax Finechem Pty Ltd., Taren Point, Australia. Sulphuric acid with concentration 95-97\%, and chloroform (purity 99.0-99.4\%, lab grade) were bought from Merck KGaA, Darmstadt, Germany.

\subsection{Preparation of flax nanocellulose fibres}

Bleached flax yarns were boiled in distilled water for $30 \mathrm{~min}$ and oven-dried to constant weight. The dried yarns were then soaked for $30 \mathrm{~min}$ in $1 \%$ (w/w) aqueous $\mathrm{NaOH}$ at $80^{\circ} \mathrm{C}$, and were washed again in running water, i.e. in an alkali-free environment. An aqueous suspension of nanocellulose fibres was prepared by acid hydrolysis for $4 \mathrm{~h}$ in $55 \mathrm{wt} \%$ sulphuric acid at $55^{\circ} \mathrm{C}$ with continuous stirring. After completion of the hydrolysis, the flask was cooled in ice-cold water. The aqueous suspension of fibres was diluted and repeatedly washed by centrifugation, then neutralised with $1.0 \mathrm{wt} \%$ aqueous $\mathrm{NaOH}$. The suspension was then frozen and freeze dried for $48 \mathrm{~h}$.

\subsection{Preparation of neat PLA and PLA composite films}

The neat PLA films were prepared by casting PLA solution in chloroform at a concentration of $5.0 \mathrm{wt} \%$. The PLA/chloroform mixtures were continuously stirred at room temperature until the pellets were fully dissolved. The composite films were obtained by mixing a $5 \mathrm{wt} \%$ suspension of $\mathrm{FC}$ in chloroform with varying proportions of the PLA solution, and stirring for $12 \mathrm{~h}$. The mixtures were then poured into Petri dishes and chloroform was allowed to evaporate at ambient temperature and pressure. The resulting films were finally oven dried for $12 \mathrm{~h}$ at $40^{\circ} \mathrm{C}$.

\subsection{Characterisation}

The structure of hydrolysed flax cellulose was investigated using transmission electron microscope (TEM - Philips CM 12, Holland). A droplet of the diluted suspension was allowed to float on and eventually flow through a copper grid covered with a carbon film. The samples were then stained by allowing the grids to float in a $2.0 \mathrm{wt} \%$ solution of uranyl acetate for $1 \mathrm{~min}$. X-ray diffraction (XRD - Bruker D 8 Advance, Germany) measurements were performed using $\mathrm{CuK}_{\alpha}$ radiation at a scan speed $0.02^{\circ} \cdot \mathrm{s}^{-1}$ from 5 to $50^{\circ}$. The tensile properties were evaluated with an Instron universal testing machine (model 5567, USA), with a $200 \mathrm{~N}$ load cell. The crosshead speed was maintained at $50 \mathrm{~mm} \cdot \mathrm{min}^{-1}$ during testing. The samples were $15 \mathrm{~mm}$ wide and $85 \mathrm{~mm}$ long, and the distance between the grips was $60 \mathrm{~mm}$. The results presented here are the average values of six individual samples. Thermal behaviour was investigated by a differential scanning calorimeter (DSC, TA Instruments Q1000, USA) using approximately $7.0 \mathrm{mg}$ of sample material. For heating-cooling-heating measurements, the following procedure was performed: isotherm at $0^{\circ} \mathrm{C}$ for $1 \mathrm{~min}$; heat at $10^{\circ} \mathrm{C} \cdot \mathrm{min}^{-1}$ from 0 to $200^{\circ} \mathrm{C}$; isotherm at $200^{\circ} \mathrm{C}$ for $1 \mathrm{~min}$; cool at $5^{\circ} \mathrm{C} \cdot \mathrm{min}^{-1}$ from 200 to $0^{\circ} \mathrm{C}$. 


\section{Results and discussion}

The TEM micrographs in Figure 1 show that sulphuric acid hydrolysis of FC caused breakdown of the fibres into rod-like fragments. The amorphous parts were selectively hydrolysed, and the crystalline parts remained unaffected. The average diameter and length for individual cellulose fibres were measured as 20 and $300 \mathrm{~nm}$, respectively. Treating cellulose with sulphuric acid involves esterification of hydroxyl groups by sulphate ions [11]. Introduction of sulphate groups along the surface of the crystallites will result in a negative charge of the surface. This anionic stabilization via the attraction/repulsion forces of the electrical double layers at the crystallites is probably the reason for the stability of the colloidal suspensions of the crystallites [12]. However, upon drying the crystalline fragments were rod-like and aggregated to some extent. The aggregation might have resulted from the formation of hydrogen bonds from the hydroxyl groups and the high surface energy of the cellulose nanofibres.

The X-ray diffraction patterns of freeze dried flax cellulose powder and non-hydrolysed flax cellulose (bleached flax yarns), shown in Figure 2, are typical cellulose I structures. The patterns of commercial microcrystalline cellulose are also shown for comparison. The crystallinity index was calculated according to Equation (1) [13]:

$$
I_{c}[\%]=\frac{I_{(200)}-I_{(a m)}}{I_{(200)}} \cdot 100
$$

where $I_{(200)}$ is the counter reading at peak intensity at a $2 \theta$ angle close to $22.8^{\circ}$ representing the crys-

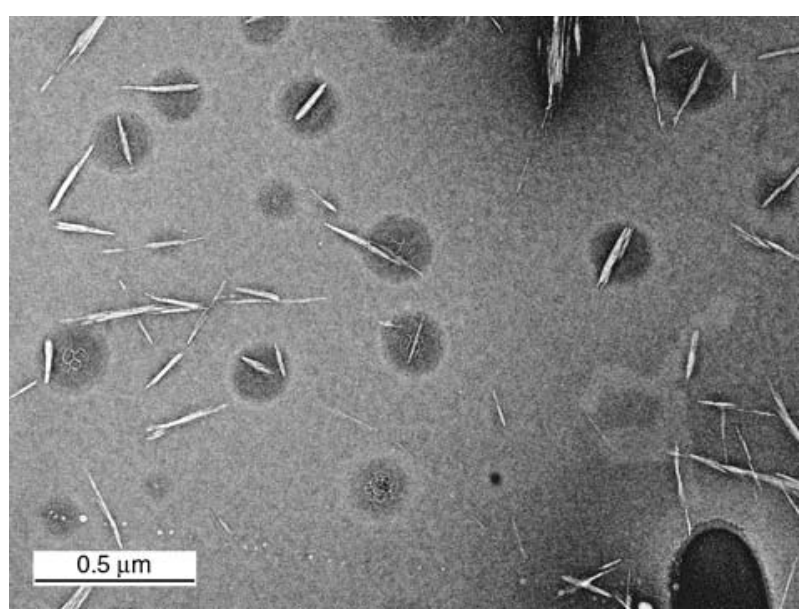

Figure 1. Transmission electron micrograph of flax cellulose nanofibres using sulphuric acid hydrolysis

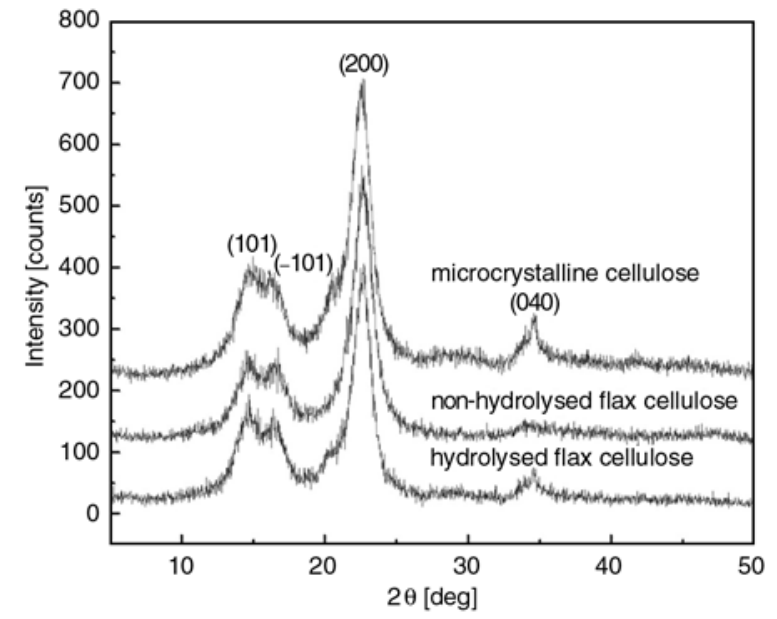

Figure 2. X-ray diffraction patterns of non-hydrolysed flax cellulose (bleached flax yarns) and freezedried flax cellulose. Commercial microcrystalline cellulose is shown for comparison.

talline part and amorphous part, and $I_{(a m)}$ is the counter reading at peak intensity at a $2 \theta$ angle around $18.6^{\circ}$ representing the amorphous part in the cellulose fibres. Use of Equation (1) from Figure 2 gives for the freeze dried flax cellulose the crystallinity index $I_{\mathrm{c}}=82 \%$, a little lower than those of non-hydrolysed flax yarns $\left(I_{\mathrm{c}}=83 \%\right)$ and commercial microcrystalline cellulose $\left(I_{\mathrm{c}}=85 \%\right)$. The lower crystallinity index of hydrolysed flax cellulose is because degradation of crystalline cellulose has probably occurred due to the hydrolysis in order to obtain isolated nanofibres [14].

Representative stress-strain curves of the pure PLA and PLA-FC composites are shown in Figure 3. From the tensile results shown in Figure 3, it is apparent that the composites show improvement in tensile modulus (measured between strains of 0.05

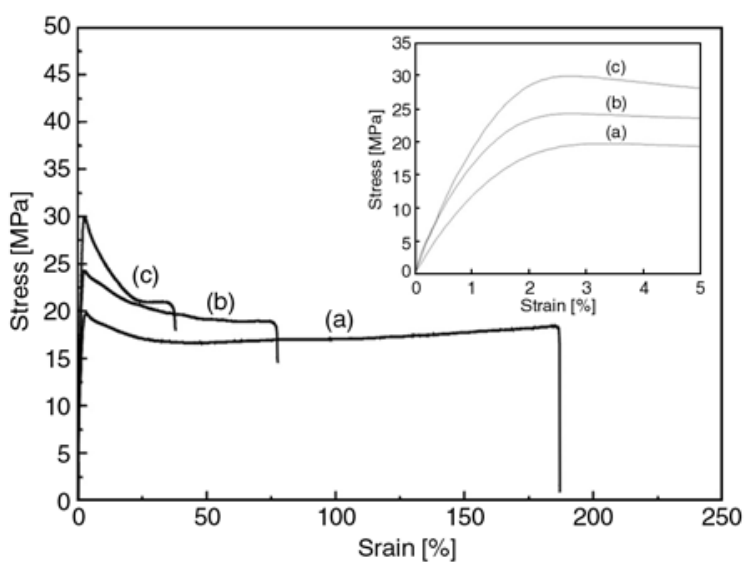

Figure 3. Stress-strain curves of pure PLA and composite films. The inset shows the stress-strain behaviour at low strains. 


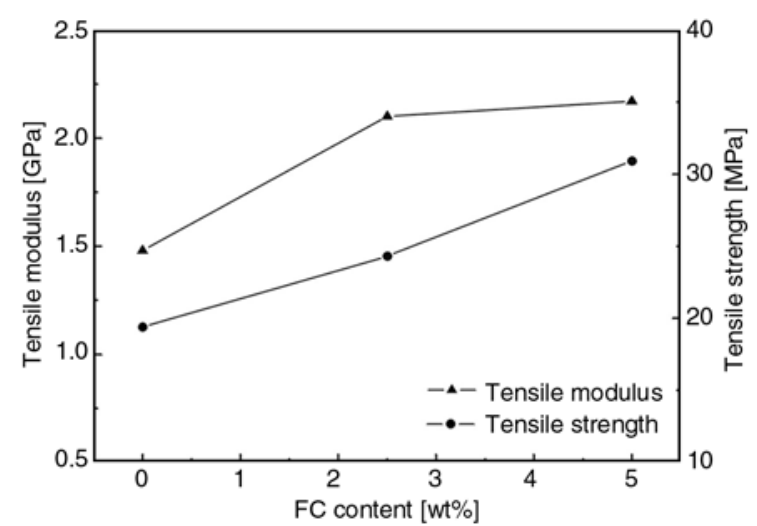

Figure 4. Tensile properties of PLA and PLA-FC composites

and $0.25 \%$ ) and tensile strength, compared to pure PLA. The tensile strength of pure PLA film and PLA films containing 2.5 and $5.0 \mathrm{wt} \% \mathrm{FC}$ was measured as $19.4,24.3$, and $30.9 \mathrm{MPa}$, respectively. Thus FC increased the tensile strength of PLA by 25 or $59 \%$ with the addition of 2.5 or $5.0 \mathrm{wt} \%$, respectively. The tensile modulus is measured as 1.48, 2.10, and 2.17 GPa for pure PLA film and PLA films with 2.5 and $5.0 \%$ FC addition, respectively. Thus FC increased the tensile modulus by 42 or $47 \%$ with the addition of 2.5 or $5.0 \mathrm{wt} \%$ respectively (Figure 4). However, the elongation at break (ductility) decreased from $194 \%$ for neat PLA film to 70 and $40 \%$ for the composites with 2.5 and $5.0 \mathrm{wt} \% \mathrm{FC}$, respectively. The composite film with $2.5 \mathrm{wt} \%$ flax cellulose combined high strength and ductility with tensile strength of $24.3 \mathrm{MPa}$ and $70 \%$ elongation at break. To investigate the effects of flax cellulose fibres on the thermal behaviours of PLA matrix, a DSC study was performed on the pure PLA and PLA-FC composite films. The thermal parameters, such as, glass transition onset temperature $\left(T_{g}\right)$, cold crystallisation onset temperature $\left(T_{c}\right)$, and melting onset temperature $\left(T_{m}\right)$ were measured from the DSC curves. The degree of crystallinity of the PLA in the composites was evaluated by the percent crystallinity of PLA in the composites, $X_{c}$, which can be calculated by Equation (2) [15]:
$X_{c}[\%]=\frac{\frac{\Delta H_{m}+\Delta H_{c}}{X_{P L A}}}{\Delta H_{m}^{c}} \cdot 100$

where $\Delta H_{m}$ and $\Delta H_{c}$ are enthalpies of fusion and crystallisation, respectively, and $\Delta H_{m}^{c}=93 \mathrm{~J} \cdot \mathrm{g}^{-1}$ is the enthalpy of fusion of $100 \%$ crystalline poly(lactic acid) [16], and $X_{\text {PLA }}$ is the weight fraction of PLA in the composite. The DSC scans (first heating scan) of pure PLA and PLA-FC composite films are depicted in Figure 5. The as-cast composite films show very similar thermal behaviour to that of neat PLA film. No obvious glass transition and crystallisation were observed upon heating up to $200^{\circ} \mathrm{C}$. The enthalpy of fusion decreases from $19.6 \mathrm{~J} \cdot \mathrm{g}^{-1}$ for pure PLA film to 19.4 and $18.9 \mathrm{~J} \cdot \mathrm{g}^{-1}$ for PLA film containing 2.5 and $5.0 \mathrm{wt} \% \mathrm{FC}$, respectively, which probably results from the lower content of PLA matrix. The calculated percent crystallinity $X_{c}$ of the three samples is almost the same, indicating that flax cellulose did not change the crystallinity of PLA during the solution casting process. The thermal parameters for the first heating run are tabulated in Table 1.

However, different thermal behaviour shown in Figure 6 was seen during the second heating run after the samples had been cooled at $5^{\circ} \mathrm{C} \cdot \mathrm{min}^{-1}$

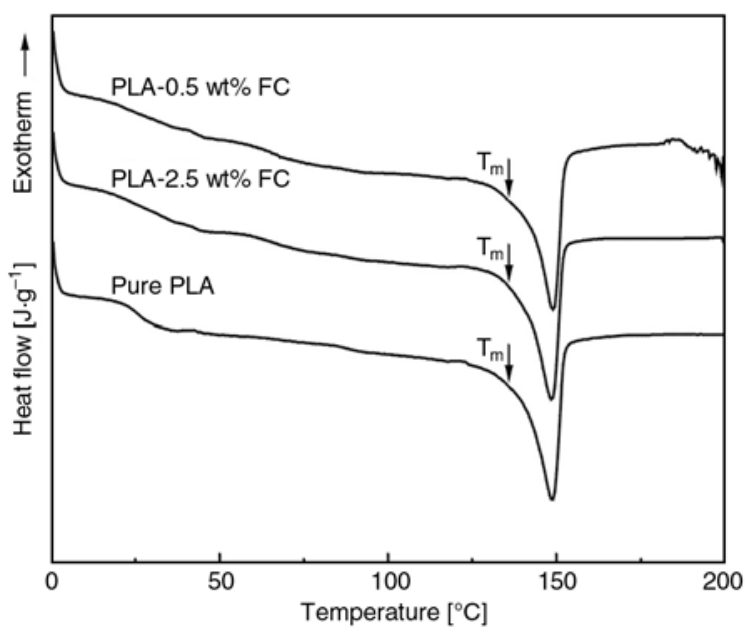

Figure 5. Thermal behaviour of pure PLA, PLA-2.5 wt $\%$ FC and PLA-5.0 wt \% FC composites during the first heating scan

Table 1. DSC results for pure PLA and PLA-FC composites from the first and second heating runs

\begin{tabular}{|c|c|c|c|c|c|c|c|c|c|}
\hline \multirow{2}{*}{ Composition } & \multirow{2}{*}{$\begin{array}{c}\mathbf{T}_{\mathbf{g}} \\
{\left[{ }^{\circ} \mathbf{C}\right]}\end{array}$} & \multirow{2}{*}{$\begin{array}{c}\mathbf{T}_{\mathbf{c}} \\
{\left[{ }^{\circ} \mathbf{C}\right]}\end{array}$} & \multicolumn{2}{|c|}{$\mathbf{T}_{\mathrm{m}}\left[{ }^{\circ} \mathbf{C}\right]$} & \multicolumn{2}{|c|}{$\Delta \mathbf{H}_{\mathrm{m}}\left[\mathrm{J} \cdot \mathrm{g}^{-1}\right]$} & \multirow{2}{*}{$\underset{\left[\mathbf{J} \cdot \mathbf{g}^{-1}\right]}{\Delta \mathbf{H}_{\mathbf{c}}}$} & \multicolumn{2}{|c|}{$\mathbf{X}_{\mathrm{c}}[\%]$} \\
\hline & & & $1^{\text {nd }}$ & $2^{\text {nd }}$ & $1^{\text {nd }}$ & $2^{\text {nd }}$ & & $1^{\text {nd }}$ & $2^{\text {nd }}$ \\
\hline Neat PLA & 56.0 & 117.3 & 139.9 & 141.8 & 19.6 & 0.16 & 0.14 & 21.1 & 0.0 \\
\hline PLA- $2.5 \mathrm{wt} \%$ FC & 55.5 & 112.1 & 140.1 & 142.2 & 19.4 & 1.81 & 1.42 & 21.4 & 0.4 \\
\hline PLA-5.0 wt $\%$ FC & 55.3 & 108.7 & 141.2 & 142.4 & 18.9 & 2.81 & 1.90 & 21.4 & 1.0 \\
\hline
\end{tabular}




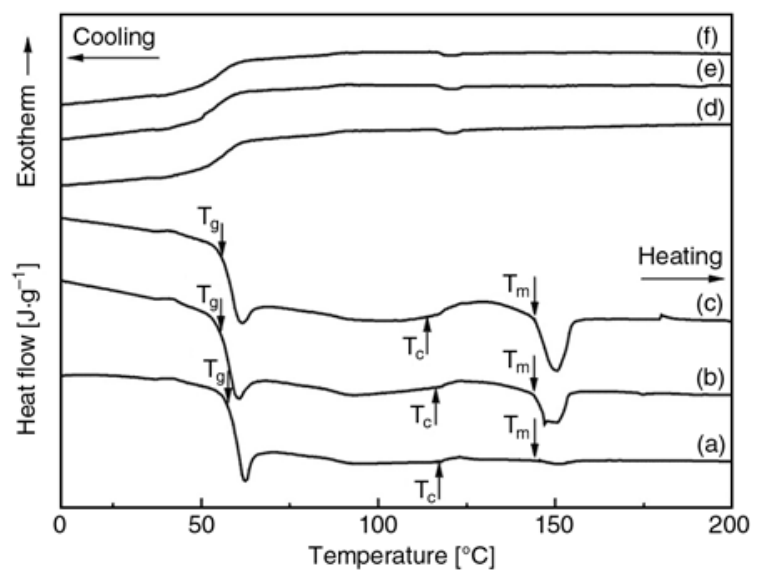

Figure 6. The cooling behaviour of pure PLA (a), PLA$2.5 \mathrm{wt} \%$ FC (b) and PLA-5.0 wt\% FC (c) composites and heating behaviour of PLA $(\mathrm{d})$, PLA-2.5 wt\% FC (e) and PLA-5.0 wt\% FC (f) during the second heating scan

from $200^{\circ} \mathrm{C}$. The three samples show a well defined glass transition, 'cold' crystallisation and melting, which are different in several respects from the thermal behaviour in the first heating scan. The composite films show a broader cold crystallisation temperature range and lower cold crystallisation onset temperature than those of the pure PLA film. The thermal parameters of this heating run are listed in Table 1. The glass transition onset temperature $\left(T_{g}\right)$ of the three samples is almost same, between $55-56^{\circ} \mathrm{C}$. The enthalpy of crystallisation and fusion of PLA-FC increased with addition of flax cellulose to PLA matrix, compared to pure PLA film. The composite films show higher percent crystallinity of 0.4 and $1.0 \%$ for the films containing 2.5 and $5.0 \mathrm{wt} \% \mathrm{FC}$, respectively. The onset crystallisation temperature decreased to 112 and $109^{\circ} \mathrm{C}$ for the composite films with 2.5 and $5.0 \mathrm{wt} \% \mathrm{FC}$, respectively, compared with $117^{\circ} \mathrm{C}$ for the pure PLA film. This behaviour indicates that the flax cellulose nano-fibre can induce crystal nucleation of the PLA polymer, which implies that this flax cellulose material can probably be used as a nucleating agent for PLA.

The different effect of flax cellulose on the thermal behaviour of PLA matrix during the same heating process is probably related to the different microstructures of the starting sample before heating. From Figure 6, the structure of pure PLA film is almost fully amorphous, because the areas under the cold crystallisation exotherm and melting endotherm are very similar, and the enthalpy of fusion is very small. Moreover, the originally cast films for the first heating run were crystalline because no cooling was applied to the casting process, providing enough time for crystallisation of dissolved polymer, while the films after cooling at $5^{\circ} \mathrm{C} \cdot \mathrm{min}^{-1}$ from $200^{\circ} \mathrm{C}$ for the second heating run were almost amorphous because the crystallisation was retarded during the cooling process, which is shown in Figure 6 that there is no obvious crystallisation from the cooling curves. This indicates that the influence of flax cellulose on the thermal behaviour of PLA matrix is related to the sample's history.

\section{Conclusions}

A suspension of cellulose nanocrystals was prepared from flax yarns by acid hydrolysis and used as reinforcement in a PLA matrix for preparation of composite films by a convenient casting method. The nanocrystal contents used were 2.5 and $5.0 \mathrm{wt} \%$. The tensile strength increased 25 and $59 \%$ for PLA composites containing 2.5 and $5.0 \mathrm{wt} \%$ flax cellulose, respectively, while the tensile modulus increased 42 and $47 \%$ for those composites. However, the elongation at break (ductility) decreased from $194 \%$ for neat PLA film to 70 and $40 \%$ for the composites with 2.5 and $5.0 \mathrm{wt} \% \mathrm{FC}$, respectively. The composite film with $2.5 \mathrm{wt} \%$ flax cellulose combines high strength and ductility with tensile strength of $24.3 \mathrm{MPa}$ and $70 \%$ elongation at break. Flax cellulose appeared to facilitate nucleation and subsequent crystallisation of PLA more effectively in the amorphous composites than in crystalline composites.

\section{Acknowledgements}

The authors are thankful for the financial support provided by the University of Auckland.

\section{References}

[1] Kale G., Auras R., Singh S. P.: Degradation of commercial biodegradable packages under real composting and ambient exposure conditions. Journal of Polymers and the Environment, 14, 317-334 (2006). DOI: $\underline{10.1007 / \mathrm{s} 10924-006-0015-6}$ 
[2] Petersen K., Nielsen P., Olsen M.: Physical and mechanical properties of biobased materials-starch, polylactate and polyhydroxybutyrate. Starch, 53, 356-361 (2001).

DOI: $\underline{10.1002 / 1521-379 X(200108) 53: 8<356:: A I D-}$ STAR356>3.0.CO;2-7

[3] Bastioli C.: Global status of the production of biobased packaging materials. Starch, 53, 351-355 (2001).

DOI: $10.1002 / 1521-379 X(200108) 53: 8<351::$ AIDSTAR351>3.0.CO;2-R

[4] Oksman K., Skrifvars M., Selin J-F.: Natural fibres as reinforcement in polylactic acid (PLA) composites. Composites Science and Technology, 63, 1317-1324 (2003).

DOI: $10.1016 / \mathrm{s} 0266-3538(03) 00103-9$

[5] Šturcová A., Davies G. R., Eichhorn S. J.: Elastic modulus and stress-transfer properties of tunicate cellulose whiskers. Biomacromolecules, 6, 1055-1061 (2005).

DOI: $\underline{10.1021 / \mathrm{bm} 049291 \mathrm{k}}$

[6] Cao X. D., Dong H., Li C. M.: New nanocomposite materials reinforced with flax cellulose nanocrystals in waterborne polyurethane. Biomacromolecules, 8 , 899-904 (2007).

DOI: $\underline{10.1021 / \mathrm{bm} 0610368}$

[7] Azizi Samir M. A. S., Alloin F., Dufresne A.: Review of recent research into cellulose whiskers, their properties and their application in nanocomposite field. Biomacromolecules, 6, 612-626 (2005).

DOI: $10.1021 / \mathrm{bm} 0493685$

[8] Lagaron J. M., Catalá R., Gavara R.: Structural characteristics defining high barrier polymeric materials. Materials Science and Technology, 20, 1-7 (2004). DOI: $10.1179 / 026708304225010442$

[9] Khalil H. P. S. A., Shahnaz Sharifah B. S., Ratnam M. M., Ahmad F., Nik Fuaad N. A.: Recycled polypropylene (RPP) wood saw dust (WSD) composites-Part1: The effect of different filler size and filler loading on mechanical and water absorption properties. Journal of Reinforced Plastics and Composites, 25, 12911303 (2006).

DOI: $10.1177 / 0731684406062060$
[10] Alexandra M., Dubois P.: Polymer-layered silicate nanocomposites: Preparation, properties and uses of a new class of materials. Materials Science and Engineering: R: Reports, 28, 1-63 (2000).

DOI: $\underline{10.1016 / \mathrm{S} 0927-796 \mathrm{X}(00) 00012-7}$

[11] Yao S. J.: Sulfation kinetics in the preparation of cellulose sulphate. Chinese Journal of Chemical Engineering, 7, 47-55 (1999).

[12] Marchessault R. H., Morehead F. F., Koch M. J.: Some hydrodynamic properties of neutral suspensions of cellulose crystallites as related to size and shape. Journal of Colloid Science, 16, 327-344 (1961). DOI: $10.1016 / 0095-8522(61) 90033-2$

[13] Segal S., Creely J. J., Martin A. E., Conrad C. M.: Am empirical method for estimating the degree of crystallinity of native cellulose using the X-ray diffractometer. Textile Research Journal, 29, 786-794 (1959). DOI: $10.1177 / 004051755902901003$

[14] Bondeson D., Mathew A., Oksman K.: Optimization of the isolation of nanocrystals from microcrystalline cellulose by acid hydrolysis. Cellulose, 13, 171-180 (2006). DOI: $10.1007 / \mathrm{s} 10570-006-9061-4$

[15] Ke T. Y., Sun X. Z.: Physical properties of poly (lactic acid) and starch composites with various blending ratios. Cereal Chemistry, 77, 761-768 (2000). DOI: 10.1094/CCHEM.2000.77.6.761

[16] Fisher E. W., Sterzel H. J., Wegner G.: Investigation of the structure of solution grown crystals of lactide copolymers by means of chemical reactions. Colloid and Polymer Science, 251, 980-990 (1973). DOI: $\underline{10.1007 / \mathrm{BF} 01498927}$ 